\title{
Measurement uncertainty caused by distance errors during in situ tests of wind turbines
}

\author{
Cornelia Reschka $^{1}$, Sebastian Koj ${ }^{2}$, Sven Fisahn ${ }^{1}$, and Heyno Garbe ${ }^{1}$ \\ ${ }^{1}$ Institute of Electrical Engineering and Measurement Technology, Leibniz University Hannover, Hannover, 30167, Germany \\ ${ }^{2}$ IAV GmbH, EMC \& Antenna, Gifhorn, 38518, Germany \\ Correspondence: Cornelia Reschka (reschka@geml.uni-hannover.de)
}

Received: 14 February 2019 - Revised: 21 May 2019 - Accepted: 7 June 2019 - Published: 19 September 2019

\begin{abstract}
During the assessment of the electromagnetic emissions of wind turbines (WTs), the aspects of measurement uncertainty must be taken into account. Therefore, this work focuses on the measurement uncertainty which arises through distance errors of the measuring positions around a WT.

The measurement distance given by the corresponding standard is $30 \mathrm{~m}$ with respect to the WT tower. However, this determined distance will always differ e.g. due to unevenness of the surrounding ground, leading to measurement uncertainties. These uncertainties can be estimated with the knowledge of the electromagnetic field distribution. It is assumed in standard measurements, that the electromagnetic field present is a pure transversal electromagnetic field (far field). Simulations of a simplified WT model with a hub height of $100 \mathrm{~m}$ shows that this assumption is not effective for the whole frequency range from $150 \mathrm{kHz}$ to $1 \mathrm{GHz}$. For frequencies below $3 \mathrm{MHz}$ the field distribution is monotonically decreasing with the distance from the WT since it behaves like an electrical small radiator. Whereas for frequencies above $3 \mathrm{MHz}$, where the investigated model forms an electrical large radiator, the field distribution becomes more complex and the measurement uncertainty of the field strength at the observation point increases. Therefore, this work focuses on investigations where the near field becomes a far field. Based on the simulation results, a method for minimizing the uncertainty contribution caused by distance errors is presented. Therefore, advanced measurement uncertainty during in situ test of WTs can be reduced.
\end{abstract}

\section{Introduction and motivation}

Every electrical system emits electromagnetic fields. Since these fields could disturb or harm other electrical systems, given limits have to be met. In the case of wind turbines (WTs), these limits are defined in the CISPR 11 (CISPR 11, 2015). For reproducible measurement results, not only are the limits defined, but also the measurement positions and frequency ranges. These definitions are further specified in a technical guideline (FGW/TR 9, 2016). For the measurement a minimum of 4 measuring positions at a distance of $r_{30}=30 \mathrm{~m}$ to the tower of the WT as shown in Fig. 1 are required. The magnetic field strength has to be measured in the CISPR Band B from $150 \mathrm{kHz}$ to $30 \mathrm{MHz}$ by using a loop antenna, the electric field strength shall be measured in the CISPR Bands C and D from $30 \mathrm{MHz}$ to $1 \mathrm{GHz}$ with a logarithmic periodic dipole antenna (LPDA) or a biconical antenna. A detailed description can be found in CISPR 11 (2015), FGW/TR 9 (2016) and Koj et al. (2018). However, without the knowledge of the measurement uncertainty, a serial release of WTs is not possible and therefore, the assessment of a WT is very time consuming and expensive.

When measuring the electromagnetic fields, one has to keep in mind, that every measuring result is afflicted by an expanded uncertainty. Therefore, the standard uncertainty has to be determined according to the "Guide to the Expression of Uncertainty" (GUM, 2008). To calculate the standard uncertainty, information about the uncertainty contributions are needed which had to be characterised for the in situ measurement. In previous works the contribution to the measurement uncertainty of the wind and of the undefined ground had already been investigated (Koj et al., 2018). In this contribution the influence of the uncertainty of the distance is presented. 


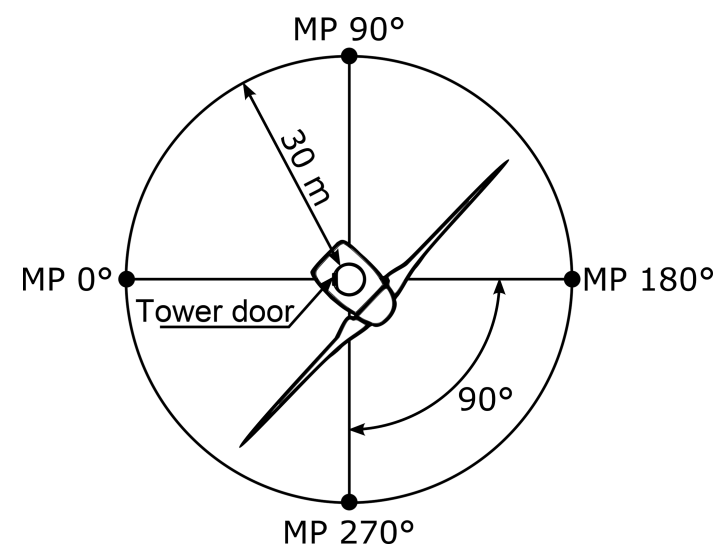

Figure 1. Measuring positions according to FGW/TR 9 (2016).

Since WTs are tested in situ, there are various causes for the uncertainty of the distance. WTs are most often located in areas used for agriculture, the ground around the WT is uneven. This leads to an inaccurate placement of the antenna due to unsuitable ground at the determined measuring position or because of inaccuracies while measuring the distance to the WT due to the given circumstances. Furthermore, the measuring equipment itself can be the cause of some inaccuracies, e.g. the antenna phase centre of a logarithmic periodic antenna that is wandering with respect to the frequency. All these factors add up to the measurement uncertainty. This aspect is investigated in this work.

Therefore, in Sect. 2 the limits for the far field are considered for electrical small and for electrical large radiators. Following, in Sect. 3 the field distribution around a wind turbine is analysed based on simulation results in hindsight of the influence of the distance uncertainty to the WT. Thereafter, a method to minimize this uncertainty contribution is presented in Sect. 4. The conclusion in Sect. 5 gives a short summary of the most important insights of this contribution.

\section{Consideration of the far field region}

As mentioned before, the frequency range for the measurement stretches from $150 \mathrm{kHz}$ to $1 \mathrm{GHz}$ and has to be measured at a distance of $r=30 \mathrm{~m}$. To assess the electromagnetic emissions, the limits given in CISPR 11 (2015) are compared with the measured field strengths. To ensure the replicability of the measurement results, it is desirable to measure the field strengths in the so-called far field region. As commonly known, the far field only has transversal components, no longitudinal components, and the field impedance is the wave impedance of free space $Z_{0}=120 \pi \Omega \approx 377 \Omega$. Various authors (Balanis, 2005; Kark, 2017; Gustrau, 2013) state that the far field of an Hertzian dipole starts at a distance of

$r \geq 2 \lambda$.

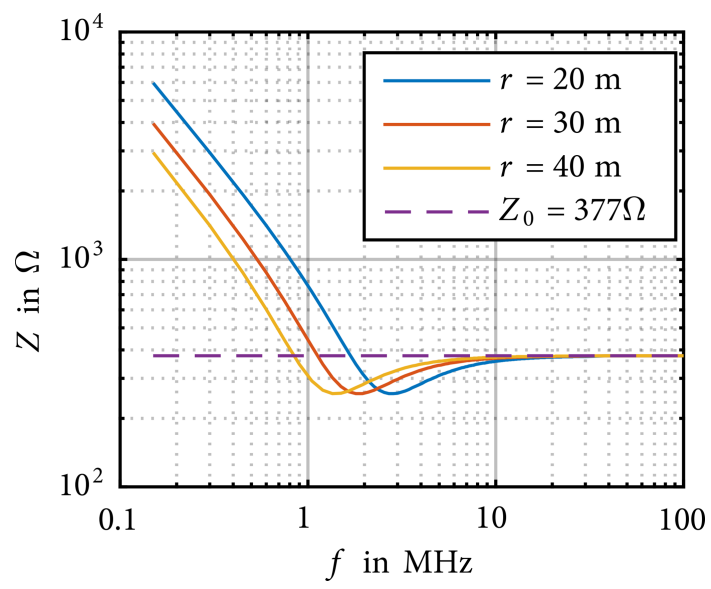

Figure 2. Field impedance of a Hertzian dipole for different distances (Koj, 2019).

Because of this, only frequencies above $20 \mathrm{MHz}$ are assumed to be measured at the distance of $30 \mathrm{~m}$ in the far field. To investigate this assumption, a simulation model with an elementary electrical dipole was built with the help of the field solver FEKO from Altair Engineering GmbH (2015).

Figure 2 shows the calculated wave impedance for distances of 20,30 and $40 \mathrm{~m}$, as well as the wave impedance of free space $Z_{0}$ over the frequency range from $150 \mathrm{kHz}$ to $100 \mathrm{MHz}$. It is evident, that the field impedance gets closer to the free space wave impedance at about $2 \mathrm{MHz}$ for $30 \mathrm{~m}$ distance and reaches the same values roughly in the range of 10 to $20 \mathrm{MHz}$. Since the wave length of $10 \mathrm{MHz}$ is $30 \mathrm{~m}$, the point of investigation is at a distance of one wavelength.

These results apply to electric elementary radiators and can also be used for small electric radiators. A radiator is electric small if the largest dimension $L_{\max }$ of the radiator is less than the wavelength $\lambda$, according to Kark (2017)

$L_{\max } \leq \lambda$.

Thus WTs with tower heights of $h_{\mathrm{T}} \geq 100 \mathrm{~m}$ have to be regarded as electrical large radiators from about $3 \mathrm{MHz}$. For such, the far field distance can be assumed to be

$r \geq \frac{2 L_{\max }^{2}}{\lambda} \geq 2 \lambda$

according to Balanis (2005), Kark (2017).

With a tower height of $h_{\mathrm{T}}=100 \mathrm{~m}$ and the maximum frequency of $1 \mathrm{GHz}$ a distance of $r=66.7 \mathrm{~km}$ would be needed. A measurement at this distance is not logistically possible, neither is a clear allocation of the measurement results to a certain WT possible. However, Laybros and Combes (2004) give the far field distance for the limiting case

$L_{\max }=\lambda$

with

$r>12 \lambda$ 


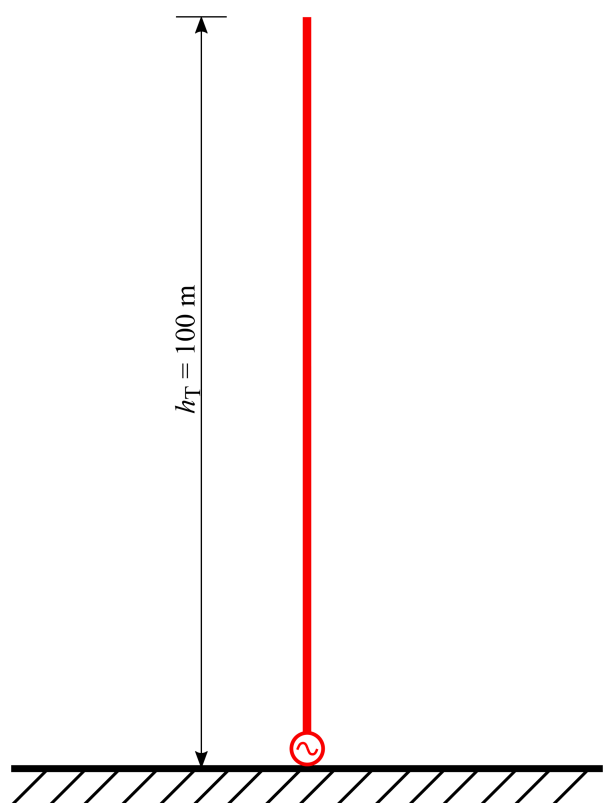

Figure 3. Simulation model of a monopole fed at the bottom over PEC.

Table 1. Investigated distances $r$ between the WT model and the observation point.

\begin{tabular}{ll}
\hline Distance $r$ & Comment \\
\hline $30 \mathrm{~m}$ & CISPR 11 requirement \\
$100 \mathrm{~m}$ & according to Eq. (4) \\
$300 \mathrm{~m}$ & according to Eq. (3) \\
$1200 \mathrm{~m}$ & according to Eq. (5) \\
\hline
\end{tabular}

which results in a distance of $r=1200 \mathrm{~m}$.

With these different definitions of the far field distance, a closer investigation for the case of a WT is done. Therefore, various distances are examined.

In order to investigate which of those definitions best suits the WT, a simulation model is built to investigate four different distances. The model shown in Fig. 3 consists of a monopole with the length $h_{\mathrm{T}}=100 \mathrm{~m}$ feed at the bottom via a voltage source. The whole model is placed over a perfect electrically conducted (PEC) ground plane. Using this model the electrical field $\boldsymbol{E}$ and the magnetic field $\boldsymbol{H}$ are calculated in varying distances $r$ between the monopole and the observation point (OP). Whereby for CISPR Band B the $\mathrm{OP}$ is placed one meter above PEC and for CISPR Band C and $\mathrm{D}$ two meters above PEC. The investigated distances are listed in Table 1. With the calculated field strengths the wave impedance is obtained according to

$Z_{\mathrm{WT}}=\frac{|\boldsymbol{E}|}{|\boldsymbol{H}|}$.

In Fig. 4 the results are shown over the frequency range from $150 \mathrm{kHz}$ to $30 \mathrm{MHz}$ and in Fig. 5 from $30 \mathrm{MHz}$ to $1 \mathrm{GHz}$.

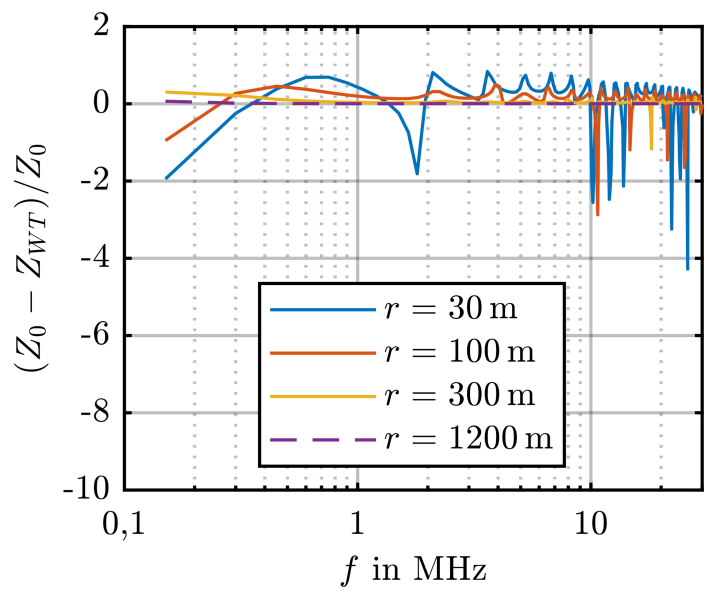

Figure 4. Field impedance of a monopole over PEC for varying distances, frequency range from $150 \mathrm{kHz}$ to $30 \mathrm{MHz}$.

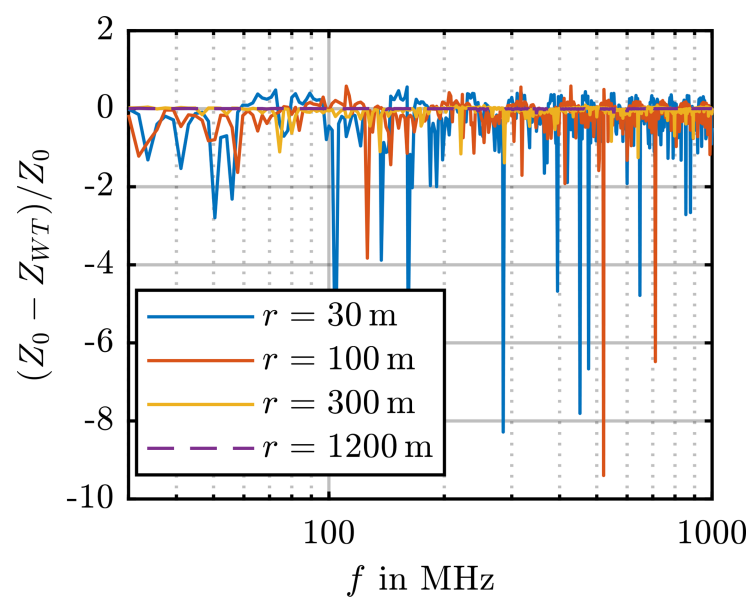

Figure 5. Field impedance of a monopole over PEC for varying distances, frequency range from $30 \mathrm{MHz}$ to $1 \mathrm{GHz}$.

The coloured lines represent the different distances and additionally, the line for the distance of $r=1200 \mathrm{~m}$ is dashed. For better comparison, all results are normalised to the free space wave impedance with

$\frac{Z_{0}-Z_{\mathrm{WT}}}{Z_{0}}$.

When the field impedance $Z_{\mathrm{WT}}$ reaches the free space wave impedance $Z_{0}$, the value will be zero. However, when the result of Eq. (7) is negative, it is a high impedance field and therefore the WT can be seen as an electrical field radiator. When the field impedance is positive, it is a low impedance field and therefore the source behaves as a magnetic field radiator. This recognition helps to identify the cause of the fields, i.e. the source of the interference. A high impedance field must therefore be caused by a voltage, a low impedance field by a current. 


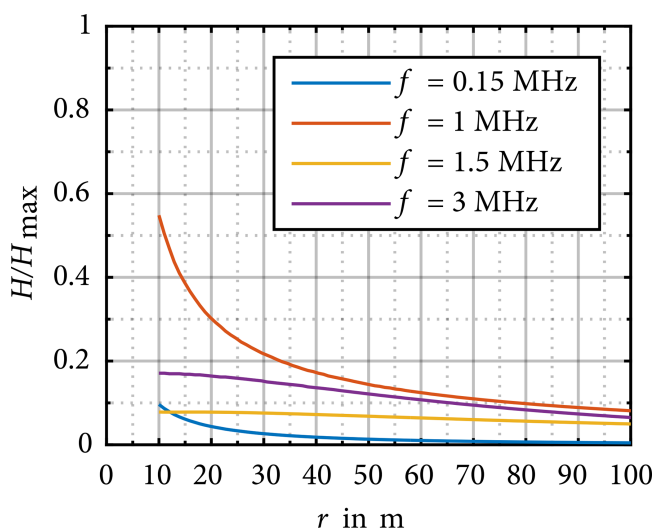

Figure 6. Magnetic field strength over the distance for discrete frequencies up to $3 \mathrm{MHz}$ (Koj, 2019).

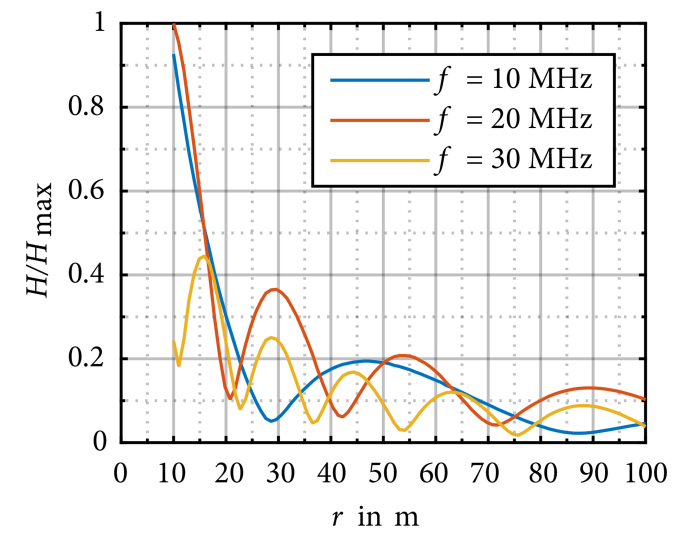

Figure 7. Magnetic field strength over the distance for discrete frequencies above $3 \mathrm{MHz}$ (Koj, 2019).

Figures 4 and 5 show that for the distance $r=30 \mathrm{~m}$ given by the CISPR 11, the far field conditions for all three CISPR Bands cannot be reached. The orange line, which represents the distance $r=100 \mathrm{~m}$, shall reach the free space wave impedance at $3 \mathrm{MHz}$, but it is clear to see, that the line does not come close to zero, which means, that the free space wave impedance is not reached as expected. The yellow line, representing the distance $r=300 \mathrm{~m}$, shall reach the far field at 4.5 MHz. The line comes close to zero between 1 and $7 \mathrm{MHz}$, however, the field impedance shows greater differences to the free space wave impedance for higher frequencies. As mentioned previously, the distance $r=1200 \mathrm{~m}$ is represented by the purple dashed line. It's clear to see, that for this distance the far field conditions are reached for the whole frequency range. This leads to the conclusion that a WT with a tower height $h_{\mathrm{T}}=100 \mathrm{~m}$ can be examined in the far field at a distance of $r=1200 \mathrm{~m}$. The tower height has to be seen as the lower limit for electrical large radiators according to Eq. (5) and therefore the far field distance of a WT can be given with

$r=12 \lambda=12 h_{\mathrm{T}}$.

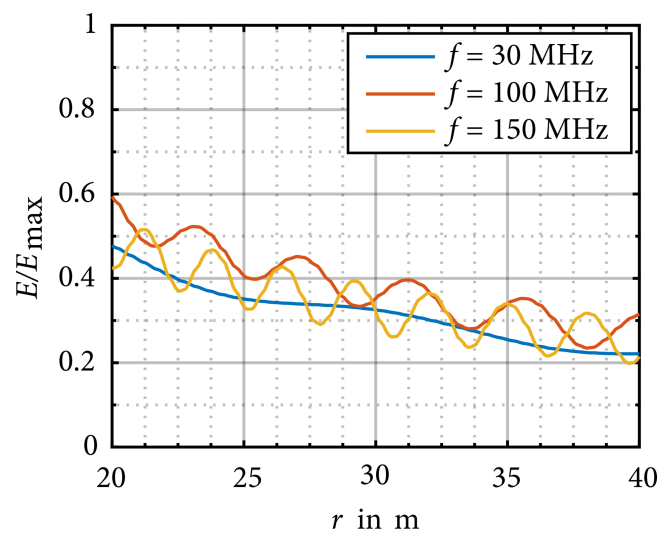

Figure 8. Electric field strength over the distance for discrete frequencies up to $150 \mathrm{MHz}$ (Koj, 2019).

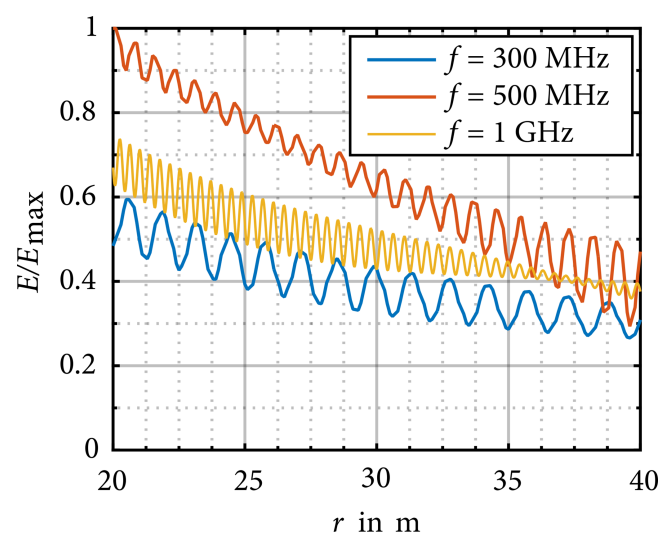

Figure 9. Electric field strength over the distance for discrete frequencies above $150 \mathrm{MHz}$ (Koj, 2019).

In summary, it can be said that the measurements of the magnetic and electric field strength taking place at the normative required distance $r_{30}=30 \mathrm{~m}$, are not in the far field at all required frequencies. Therefore, the need for the knowledge of the field distribution around the measuring position arises.

\section{Field distribution around a wind turbine and uncertainty contribution}

In order to determine the uncertainty contribution of the distance error as described previously, the field distribution around a WT is investigated by the model shown in Fig. 3. In contrast to the previous simulations, now discrete frequencies are investigated over a varying distance $r$. The distributions of the magnetic field are shown in Figs. 6 and 7 for the distance $10 \mathrm{~m} \leq r \leq 100 \mathrm{~m}$. The results are normalized to the maximal magnetic field strength $H_{\max }$. Figure 6 shows the field distribution at the frequencies $150 \mathrm{kHz}, 1 \mathrm{MHz}$, $1.5 \mathrm{MHz}$ and $3 \mathrm{MHz}$. It can be seen that the field strength is decreasing monotonically for frequencies below $3 \mathrm{MHz}$, 
thus the model is an electrical small radiator. The simulation results for the frequencies 10,20 and $30 \mathrm{MHz}$ are shown in Fig. 7. At those frequencies the model has to be seen as electrical large and the field distribution is not decreasing monotonically. Furthermore, the phenomena of standing waves can be observed due to reflections on the PEC ground.

In order to investigate the distribution of the electric field strength, simulations for distances $20 \mathrm{~m} \leq r \leq 40 \mathrm{~m}$ are calculated. The results are shown in Figs. 8 and 9 for different frequencies between $30 \mathrm{MHz}$ and $1 \mathrm{GHz}$, whereby the results of the electric field strength are normalised to its maximum $E_{\max }$. In this frequency range the WT also behaves like an electrical large radiator and standing waves occur in the electric field distribution similar to Fig. 7. The standing wave can be characterised by its local minima and maxima. The distance between the maxima decreases with increasing frequency.

With the knowledge of the field distribution, the influence of the distance error on the measurement uncertainty of the field strength can be explained. For example, the electric field strength distribution at $300 \mathrm{MHz}$ in Fig. 9 shows a local maximum at the distance $r_{30}=30 \mathrm{~m}$. In the case of the antenna being located with a distance error $\Delta r$ near $r_{30}$, a different field value will be measured.

If a logarithmic periodic dipole antenna (LPDA) is used for the measurement of the electric field strength, a distance error of $\Delta r= \pm 0.25 \mathrm{~m}$ caused by wandering phase centre along LPDA can be assumed. Finally, the resulting field uncertainty $\Delta F$ caused by $\Delta r$ can be written as

$\Delta F=20 \log \left(\frac{F\left(r_{30}+\Delta r\right)}{F\left(r_{30}\right)}\right)$.

Using Eq. (9) and the simulated field distributions, the resulting field uncertainty can be calculated for each frequency range, as shown in Figs. 10 and 11. Figure 10 shows, that in the frequency range below $10 \mathrm{MHz}$, the expected uncertainty does not exceed $\pm 1 \mathrm{~dB}$. However, for higher frequencies uncertainty values of up to $\pm 6 \mathrm{~dB}$ are possible as seen in Fig. 11.

Since the wavelength decreases with increasing frequency, the error made becomes greater with increasing frequency. Therefore, a way to minimize the uncertainty contribution caused by distance error is needed and is treated in the following section.

\section{Method to minimize the uncertainty contribution}

The goal of this chapter is to define a method which allows a reduction of the measurement uncertainty described above, the influence of the frequency and of the tower height $h_{\mathrm{T}}$ on the field distribution is investigated. Figures 7, 8 and 9 show that the distance between two maxima of each field distribution decreases with increasing frequency. Figure 12 shows the magnetic field distribution at $30 \mathrm{MHz}$ for different tower

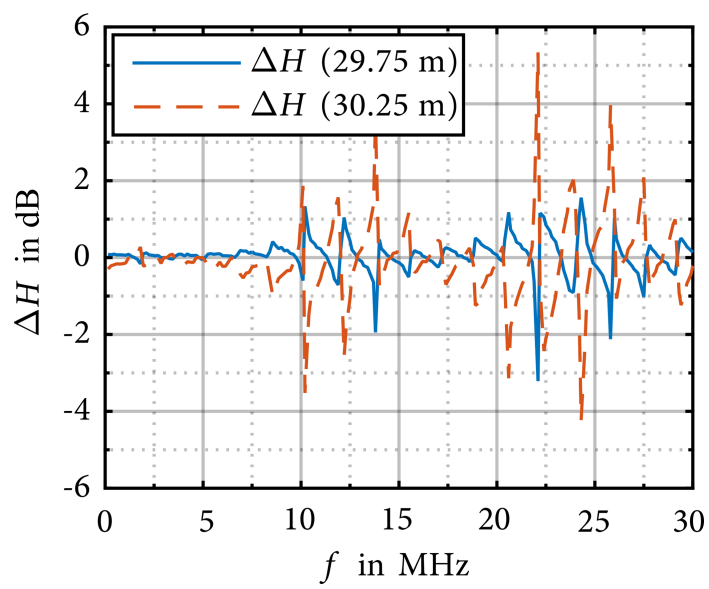

Figure 10. Uncertainty contribution caused by distance errors for the magnetic field strength (Koj, 2019).

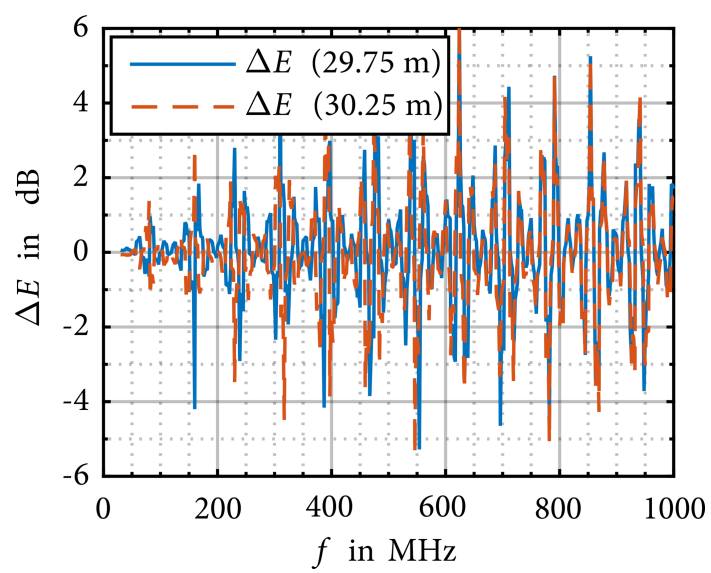

Figure 11. Uncertainty contribution caused by distance errors for the electric field strength (Koj, 2019).

heights. It can be seen that the location of each local maxima depends on the tower height. Furthermore, it is seen that the local maxima of the field distribution decreases with the distance $r$ according to $1 / r$.

Figure 13 shows the magnetic field distribution at $30 \mathrm{MHz}$ for a WT model with $h_{\mathrm{T}}=100 \mathrm{~m}$ for distances of $10 \mathrm{~m} \leq r \leq$ $100 \mathrm{~km}$. For a better presentation, the distance $r$ is given logarithmically. It can be seen, that the local maxima occur till $r \approx 1 \mathrm{~km}$ and decrease with $1 / r$. For distances $r>1 \mathrm{~km}$ the field distribution is falling monotonically and follows $1 / r$ for distances above $10 \mathrm{~km}$. Far field conditions can be expected at those distances.

The idea of reducing the influence of the distance errors and the measurement uncertainty of the magnetic and electric field strength is based on the $1 / r$ dependence of the local maxima of each field distribution. Therefore, the field distribution should be scanned near the measuring position $r_{30}$ along the distance $r$ to ensure that the maximum field strength $F_{\max }$ is measured. Here, the distance between the 


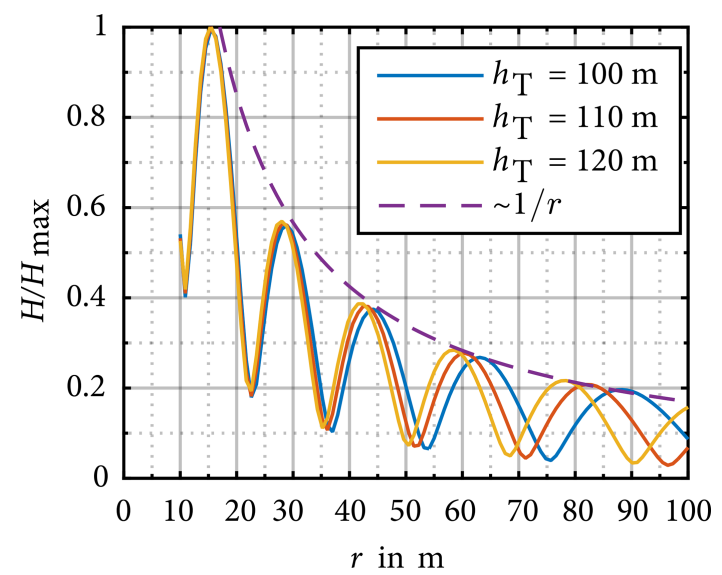

Figure 12. Field distribution for different tower heights at $30 \mathrm{MHz}$ (Koj, 2019).

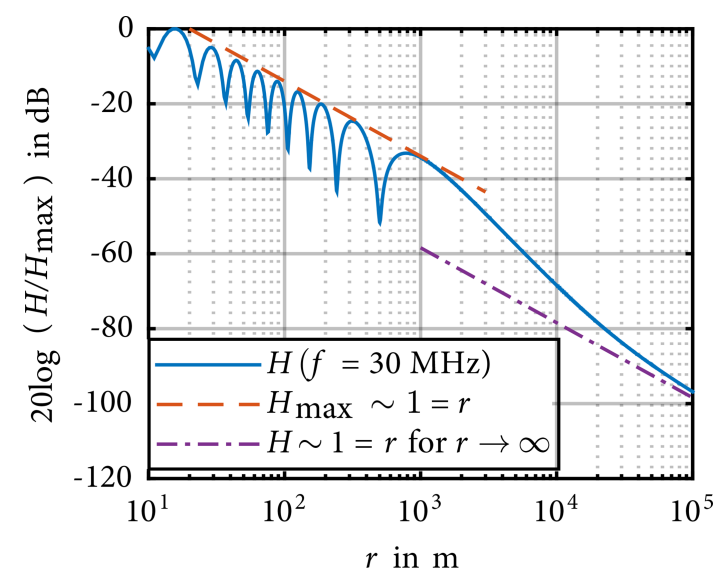

Figure 13. Field distribution for $30 \mathrm{MHz}$ at a tower height $h_{\mathrm{T}}=100 \mathrm{~m}(\mathrm{Koj}, 2019)$.

WT tower and the position of the local field maximum $r_{\mathrm{LM}}$ has to be determined as well. Using both the measured maximum field strength $F_{\max }$ and the corresponding distance $r_{\mathrm{LM}}$, the field strength $F_{30}$ at the normative required distance $r_{30}=30 \mathrm{~m}$ can be calculated using $1 / r$ dependence of the field distribution

$F_{30}=F_{\max } \frac{r_{\mathrm{LM}}}{r_{30}}$.

Finally, the calculated field strength can be compared with the given limits.

\section{Conclusion}

This work describes the contribution of distance errors to the measurement uncertainty during in situ tests of electromagnetic emissions of wind turbines (WTs). In order to investigate the far field region, a simple model of a WT is set up and numerically analysed. The simulation results show that at the normative required distance of $r=30 \mathrm{~m}$ and in the frequency range from $150 \mathrm{kHz}$ to $1 \mathrm{GHz}$ far field conditions cannot be expected.

Therefore, the field distribution near a WT is calculated. It can be shown that at those frequencies, where the WT model is an electrical small radiator, the field distribution shows a monotonically decreasing dependence on the distance $r$. For this case, the resulting measurement uncertainty of the field strength is smaller than $\pm 1 \mathrm{~dB}$. For those frequencies, at which the WT behaves like an electrical large radiator, the resulting field distribution can be described with standing waves, i.e. local minima and maxima occur. For this case, the resulting measurement uncertainty increases.

In order to reduce the measurement uncertainty at electrical large radiators, the dependence of the field distribution from the frequency and the tower height is investigated. It is shown that the distance between two maxima of the field distribution decreases with increasing frequency. The location of a local maximum also depends on the tower height. However, the simulation results show that the levels of the local maxima decrease with distance $r$ according to $1 / r$. Using this fact, a simple method to reduce the measurement uncertainty is given. For this purpose, the field strength has to be scanned near the measuring position along the distance $r$ till a local maximum is found. Finally, the measured maximum field strength must be calculated for the given normative measuring distance.

Using the presented results, the measurement uncertainty of in situ tests of radiated electromagnetic emissions from WTs can be described. With the knowledge of this uncertainty a serial release of WTs is possible. The assessment of WTs becomes more time and cost effective.

Data availability. The data are available from the corresponding author upon request.

Author contributions. All authors contributed to the design and implementation of the research, the analysis of the results and the writing of the manuscript.

Competing interests. The authors declare that they have no conflict of interest. 
Special issue statement. This article is part of the special issue "Kleinheubacher Berichte 2018". It is a result of the Kleinheubacher Tagung 2018, Miltenberg, Germany, 24-26 September 2018 .

Financial support. The publication of this article was funded by the open-access fund of Leibniz Universität Hannover.

Review statement. This paper was edited by Thorsten Schrader and reviewed by two anonymous referees.

\section{References}

Altair Engineering GmbH: Altair FEKO, Calwer Straße 7, 71034 Böblingen, Germany, available at: http://www.feko.info, http:// www.altair.com (last access: 8 July 2019), 2015.

Balanis, C. A.: Antenna Theory, 3rd Edition, John Wiley \& Sons, New Jersey, USA, 2005.

CISPR 11: Industrial, scientific and medical equipment - Radiofrequency disturbance characteristics, Limits and methods of measurement, International Electrotechnical Commission, 2015.
FGW/TR 9: Technical Guidelines for Wind Turbines (FGW Guideline) Part 9:2016, Rev. 1, Determination of High Frequency Emissions from Renewable Power Generating Units, FGW e.V. - Fördergesellschaft Windenergie und andere Dezentrale Energien, 2016.

GUM: JCGM 100: Evaluation of measurement data, Guide to the expression of uncertainty in measurement, 2008.

Gustrau, F.: Hochfrequenztechnik: Grundlagen der mobilen Kommunikationstechnik, 2nd edn., Fachbuchverlag Leipzig, München, Germany, 2013.

Kark, K. W.: Antennen und Strahlungsfelder, 6th Edition, Springer Vieweg, Wiesbaden, Germany, 2017.

Koj, S.: Messunsicherheit bei in situ Tests der elektromagnetischen Verträglichkeit von Windkraftanlagen, sierke Verlag, Göttingen, Germany, 2019.

Koj, S., Hoffmann, A., and Garbe, H.: Measurement Uncertainty of Radiated Electromagnetic Emissions in In Situ Tests of Wind Energy Conversion Systems, Adv. Radio Sci., 16, 13-22, https://doi.org/10.5194/ars-16-13-2018, 2018.

Laybros, S. and Combes, P.F.: On Radiating-Zone Boundaries of Short, $\lambda / 2$ and $\lambda$ Dipoles, IEEE Antenn. Propag. M., 46, 53-64, 2004. 